See discussions, stats, and author profiles for this publication at: https://www.researchgate.net/publication/340067514

Professional development and research are being neglected: a commentary on the 2019 RCR radiologists' supporting professional activities (SPA) survey

Article in Clinical Radiology · March 2020

DOI: 10.1016/j.crad.2020.03.001

CITATIONS

0

12 authors, including:

Owen Arthurs

Great Ormond Street Hospital for Children NHS Foundation Trust

225 PUBLICATIONS 2,272 CITATIONS

SEE PROFILE

2. Christina Messiou

The Royal Marsden NHS Foundation Trust

138 PUBLICATIONS 3,664 CITATIONS

SEE PROFILE

Some of the authors of this publication are also working on these related projects:

Project Heat illness View project

Project Body imaging View project
READS

31

Thomas C Booth

King's College London

90 PUBLICATIONS 693 CITATIONS

SEE PROFILE

Luc Bidaut

University of Lincoln

120 PUBLICATIONS 3,630 CITATIONS

SEE PROFILE 


\section{Professional development and research are being neglected: a commentary on the 2019 RCR radiologists' supporting professional activities (SPA) survey}

\section{O.J. Arthurs ${ }^{\mathrm{a}, \mathrm{b}, *}, \mathrm{~V}$. Goh $^{\mathrm{c}}$ on behalf of the RCR Academic Committee}

${ }^{a}$ Great Ormond Street Hospital for Children NHS Foundation Trust, London, WC1N 3JH, UK

${ }^{\mathrm{b}}$ NIHR Great Ormond Street Hospital Biomedical Research Centre, London, UK

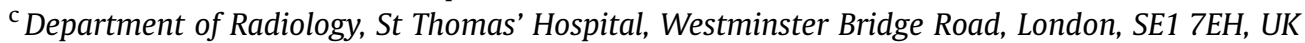

\section{ARTICLE INFORMATION}

\section{Article history:}

Received 3 March 2020

Accepted 3 March 2020

\section{Introduction}

When the National Health Service (NHS) acquired a statutory duty of care for quality in 1998, clinical governance became a mandatory and intrinsic part of modern medicine. Defined as "a framework through which NHS organisations are accountable for continuously improving the quality of their services and safe-guarding high standards of care by creating an environment in which excellence in clinical care will flourish", ${ }^{1}$ the vehicle for NHS consultants to enact clinical governance was supporting professional activity (SPA). All activities that underpin direct clinical care (DCC) are encouraged during SPA time, including professional development, research, audit, teaching, clinical management, appraisal, and job planning., ${ }^{2,3}$ Adequate time for SPAs

\footnotetext{
* Guarantor and correspondent: O. Arthurs, Great Ormond Street Hospital for Children NHS Foundation Trust, London, WC1N 3JH, UK. Tel.: +44 0207 4059200 .

E-mail address: owen.arthurs@gosh.nhs.uk (O.J. Arthurs).

RCR Academic Committee: Nigel Hoggard, Tom Booth, Christina Messiou, James The, Andrew Plumb, Luc Bidaut, Tom Turmezei, Ketan Jethwa, Philip Robinson, Margaret Hall-Craggs.
}

alongside DCC is therefore crucial for NHS consultants to maintain excellence in clinical care. ${ }^{3}$

The recently published Royal College of Radiologists (RCR) Survey on Radiologists' $\mathrm{SPA}^{4}$ has demonstrated three recurring themes, which are widely recognised to be growing concerns for our specialty.

First, NHS trusts typically offer insufficient SPA time for all supporting activities. Most consultants do not receive the 2.5 SPA per week advised by the NHS, ${ }^{2}$ with the 2018 RCR Clinical Radiology Workforce Census ${ }^{5}$ reporting that fulltime NHS consultant radiologists had an average of two SPAs (i.e., 8 hours equivalent per week) in their job plans. The latest SPA survey shows that this average has already dropped further to 1.8 SPAs, with newer consultants often getting less than two SPAs. Furthermore, $40 \%$ of respondents had only one SPA per week, and many part-time respondents (25\% of respondents) had no SPA time at all.

Second, most radiologists spend their SPA time managing clinical commitments, i.e., on activities that are actually considered to be DCC. The survey reveals that most radiologists' SPAs are spent responding to emails (due to inadequate administrative support), secondopinion work, organising individual patient care and 
logistics, and preparing for or attending clinical meetings. The minimal time spent on departmental service planning and audit, as well as job planning, only serves to reinforce the expanding administrative burden placed on the individual consultant, compounded by lack of administrative support staff.

Third, that these factors in combination severely limit the time and opportunity available for NHS clinicians to be involved in research. It is widely recognised that providing high-quality patient care is dependent upon the inherent ability of medical practitioners to participate in clinical trials, to evaluate new diagnostics, drugs, other therapeutics, and improvements in prevention, and also to participate in good research governance. ${ }^{6,7}$ Without the time to engage with and evaluate new diagnostic and treatment strategies, the NHS will fall behind other service providers in evidence-based healthcare, particularly in the most technologically driven specialties, such as clinical radiology. ${ }^{8}$

The current inadequate level of SPA time has directly contributed to a distinct lack of research involvement and leadership, as well as an inconsistent approach to quality improvement and education, at a time when advances in imaging methodology and delivery are becoming even more critical to healthcare improvements. Although there will naturally be less research capacity in the most clinically under-resourced specialties, we are particularly concerned that over half the respondents were not involved in research in any capacity over the last year, and only $10 \%$ of non-academics reported spending more than an hour each week on research. Of those who are involved, most are supporting research led by other specialties, often funded by National Institute for Health Research (NIHR) or other charitable sources, rather than leading studies themselves. The main barriers to research involvement are shortages of time (not lack of interest, funding, research infrastructure, and academic mentorship) outside of specialist centres, all further compounded by workforce shortages.

The survey's findings and its implications are symptomatic of the larger challenges the NHS faces in training, growing, and maintaining a healthy and efficient workforce. Clearly, balancing clinical service provision with year-on-year growing demands and workforce shortages is not easy. With an estimated 10\% vacancy rate in UK radiologist consultant posts, ${ }^{5}$ continuing pension taxation, and Brexit uncertainty will further heighten the challenges the NHS already has in looking after its existing workforce. ${ }^{9}$ Inadequate SPA time contributes to stress and burnout, leading to a high proportion of radiologists opting for career breaks and early retirement, which will only further exacerbate the problem. $^{3}$

We recognise that the balance between clinical service and research provision is necessarily skewed heavily and increasingly towards the former. Individual NHS trusts, the Royal Colleges, and individual consultants taking up new posts all need to be aware of the implications of reduced SPA time on the future of our speciality and delivery of patient care. Short-term solutions of reducing SPAs and reducing administrative staff may lead to short-term cost savings, but have led to a breach of the statutory duty of care for quality, and the long-term damage to the NHS will be costly to repair. In our specialty, we must ensure that a sufficient number of well-rounded radiologists can maintain the excellence in clinical care that underpins crucial NHS workflows and processes for years to come.

A co-ordinated strategy involving all stakeholders is required to resolve these critical issues. NHS trusts should commit to providing protected SPA time, financial support for academic activity within clinical radiology posts, and the development of dedicated clinical academic posts. The RCR should not endorse consultant posts with insufficient SPA time allocated. The launch of the new RCR Research Foundation will provide restructuring of the financial support available to radiology research, as well as appropriate academic mentoring for research active radiologists at all levels.

Funders such as the NIHR need to be made aware of the high barriers radiologists face in supporting and delivering imaging research. They need to recognise that radiology is a shortage speciality, especially those in lead research roles. Those that succeed in getting there frequently take less conventional routes (often involving career breaks and FRCR exam considerations), which need a more flexible, inclusive attitude and greater support than may be required for other specialties. Whilst NHS trusts and funding bodies continue to overlook (or fail to prioritise) radiology and radiologists are given neither adequate time nor opportunities to prepare or deliver radiology-led clinical research, the fundamental problems shown in this survey will continue.

Further work is clearly needed to foster a supportive research culture and provide the necessary infrastructure, tailored to the needs of clinical imaging, in order to facilitate research activity in radiology. The work of the NIHR Imaging project and NIHR Clinical Research Network imaging steering group, led by Professor Steve Smye, is a start but still a "drop in the ocean". 8 Only through high-level strategic support from all funding bodies and actively looking to promote imaging-led activities, either through infrastructure support, consultant time or scanner time, will this be tackled in the short term. Investment now will help to identify and develop high-impact research projects that will reap future rewards. Research-active trainees coming through the Radiology Academic Network for Trainees (RADIANT) and NIHR integrated academic training schemes will be looking forward to continuing and developing research activities in their consultant appointments, and we should not disappoint them.

\section{Conflict of interest}

The authors declare no conflict of interest.

\section{References}

1. Scally G, Donaldson LJ. Looking forward: clinical governance and the drive for quality improvement in the new NHS in England. BMJ 1998;317:61-5. 
2. NHS Employers. Guidance on support professional activities. 2007 https:// www.nhsemployers.org/pay-pensions-and-reward/medical-staff/ consultants-and-dental-consultants/consultants-and-dentalconsultants-tcs-handbook/documents-and-guidance. [Accessed 1 March 2020].

3. Academy of Medical Royal Colleges. Advice on supporting professional activities in consultant job planning. London: Academy of Medical Royal Colleges; 2010.

4. The Royal College of Radiologists. RCR Radiologists' support professional activities (SPA) survey. 2019 [website link here when published].

5. The Royal College of Radiologists. Clinical Radiology UK workforce census 2018 report. London: The Royal College of Radiologists; 2020 https:// www.rcr.ac.uk/clinical-radiology/service-delivery/rcr-radiologyworkforce-census. [Accessed 1 March 2020].
6. NIHR Signal: participation in health research may be linked to better care and performance. https://discover.dc.nihr.ac.uk/content/signal-000195/ participation-in-health-research-may-be-linked-to-better-care-andperformance. [Accessed 11 February 2020].

7. Boaz A, Hanne S, Jones T, et al. Does the engagement of clinicians and organisations in research improve healthcare performance: a three-stage review. BMJ Open 2015;5(12):e009415.

8. Goh V, National Institute for Health Research Clinical Research Network Imaging Network Steering Group. Royal College of Radiologists' Academic Committee. the national Institute for health research: making an impact in imaging research. Clin Radiol 2019 Mar;74:242-6.

9. Piorkowska M, Goh V, Booth TC. Post Brexit: challenges and opportunities for radiology beyond the European Union. Br J Radiol 2017 Apr;90(1072):20160852. 